Pacific Journal of Mathematics

SOLUTION OF AN INVARIANT SUBSPACE PROBLEM OF K. T. 


\title{
SOLUTION OF AN INVARIANT SUBSPACE PROBLEM OF K. T. SMITH AND P. R. HALMOS
}

\author{
Allen R. Bernstein and Abraham Robinson
}

The following theorem is proved.

Let $T$ be a bounded linear operator on an infinite-dimensional Hilbert space $H$ over the complex numbers and let $p(z) \neq 0$ be a polynomial with complex coefficients such that $p(T)$ is completely continuous (compact). Then $T$ leaves invariant at least one closed linear subspace of $H$ other than $H$ or $\{0\}$.

For $p(z)=z^{2}$ this settles a problem raised by P. R. Halmos and K. T. Smith.

The proof is within the framework of Nonstandard Analysis. That is to say, we associate with the Hilbert space $H$ (which, ruling out trivial cases, may be supposed separable) a larger space, ${ }^{*} H$, which has the same formal properties within a language $L . \quad L$ is a higher order language but ${ }^{*} H$ still exists if we interpret the sentences of $L$ in the sense of Henkin. The system of natural numbers which is associated with ${ }^{*} H$ is a nonstandard model of arithmetic, i.e., it contains elements other than the standard natural numbers. The problem is solved by reducing it to the consideration of invariant subspaces in a subspace of ${ }^{*} H$ the number of ${ }^{\circ}$ whose dimensions is a nonstandard positive integer.

\section{Introduction. We shall prove:}

MAIN THEOREM 1.1. Let $T$ be a bounded linear operator on an infinite-dimensional Hilbert space $H$ over the complex numbers and let $p(z) \neq 0$ be a polynomial with complex coefficients such that $p(T)$ is completely continuous (compact). Then $T$ leaves invariant at least one closed subspace of $H$ other than $H$ or $\{0\}$.

For $p(z)=z^{2}$ this settles Problem No. 9 raised by Halmos in [2] and there credited to K. T. Smith. For this case, a first proof was given by one of us (A.R.) while the other (A.R.B.) provided an alternative proof which extends to the case considered in 1.1. The argument given below combines the two proofs, both of which are based on Nonstandard Analysis. The Nonstandard Analysis of Hilbert space was developed previously by A.R. as far as the spectral analysis of completely continuous self-adjoint operators (compare [7]) while A.R.B. has disposed of the spectral theorem for bounded self-adjoint operators

Received July 5, 1964, and in revised form December 10, 1964. The authors acknowledge with thanks the support received from the National Science Foundation (Grant No. GP-1812). 
by the same method. The general theory will be sketched here only as far as it is required for the proof of our main theorem.

Some of our arguments are adapted from the proofs of the theorem for $p(z)=z$, i.e., when $T$ is itself completely continuous, which are due to von Neumann and Aronszajn for Hilbert space, as above, and to Aronszajn and K. T. Smith for general Banach spaces [1].

The particular version of Nonstandard Analysis which is convenient here relies on a higher order predicate language, $L$, which includes symbols for all complex numbers, all sets and relations of such numbers, all sets of such sets and relations, all relations of relations, etc. Quantification with respect to variables of all these types is permitted. Within this framework, a sequence of complex numbers, $y=s_{n}, n=$ $1,2,3, \cdots$, is given by a many-one relation $S(n, y)$ when $n$ varies over the set of positive integers, $P$. The separable Hilbert space, $H$, may then be represented as a set of such sequences (i.e., as $l_{2}$ ) while a particular operator on $H$ is identified with a relation of relations.

Let $K$ be the set of sentences formulated in $L$ which hold in the field of complex numbers, $C$. $K$ includes sentences about, or involving, the sets of real numbers and of natural numbers, since these may be regarded as subsets of the complex numbers which are named in $L$. It also includes sentences about Hilbert space as represented above.

Nonstandard Analysis is based on the fact that, in addition to $C$, $K$ possesses other models, which are proper extensions of $C$. We single out any one of them, ${ }^{*} C$, calling it the nonstandard model, as opposed to the standard model, $C$. However, ${ }^{*} C$ is a model of $K$ only if the notions of set, relations, etc. are interpreted in ${ }^{*} C$ in the sense of the higher order model theory of Henkin [3]. That is to say, the sets of sets, relations, etc., which are taken into account in the interpretation of a sentence in ${ }^{*} C$ may (and will) be proper subsets of the corresponding sets over ${ }^{*} C$ in the absolute sense. The sets, relations, etc. which are taken into account in the interpretation in ${ }^{*} C$ will be called admissible.

The basic properties and notions of Nonstandard Analysis which are expounded in [4] and [5] are applicable here. Thus, an individual of ${ }^{*} C$ (which will still be called a complex number) may or may not be an element of $C$, i.e., a complex number in the ordinary sense or standard number, briefly an S-number. Every finite complex number $a$ is infinitely close to a unique standard complex number, ${ }^{0} a$. That is to say, if $|a|$ is smaller than some real $S$-number, then there exists a complex $S$-number, ${ }^{\circ} a$, the standard part of $a$, such that $\left|a-{ }^{0} a\right|$ is smaller than all positive $S$-numbers. A number which is infinitely close to 0 is infinitely small or infinitesimal. In particular, 0 is the only $S$-number which is infinitesimal. A complex number a which is not finite, i.e., which is such that $|a|$ is greater than any $S$-number, is infinite. There exist elements of ${ }^{*} C$ which are infinite. 
Every set, relation, etc. in $C$ possesses a natural extension to ${ }^{*} C$. This is simply the set, relation, $\cdots$, in ${ }^{*} C$ which is denoted by the same symbol in $L$. At our convenience, we may, or may not, denote it by the same symbol also in our notation (which is not necessarily part of $L$ ). Thus, we shall denote the extension of the set of positive integers, $P$, to ${ }^{*} C$ by ${ }^{*} P$ but if $\sigma=\left\{a_{n}\right\}$ is a sequence of complex numbers in $C$ then we shall denote its extension to ${ }^{*} C$ still by $\sigma=\left\{a_{n}\right\}$. According to the definition of an infinite number which was given above, the infinite positive integers in ${ }^{*} C$ are just the elements of ${ }^{*} P-P$.

The following results are basic (for the proofs see [5] and [6]).

THEOREM 1.2. The sequence $\left\{a_{n}\right\}$ in $C$ converges to a limit $a$ (a an $S$-number) if and only if the extension of $\left\{a_{n}\right\}$ in ${ }^{*} C$ satisfies the condition that $\left|a-a_{n}\right|$ is infinitesimal for all infinite $n$.

THEOREM 1.3. Let $\left\{a_{n}\right\}$ be an admissible sequence in ${ }^{*} C$ such that $a_{n}$ is infinitesimal for all finite $n$. Then there exists an infinite positive integer $\omega\left(\right.$ i.e., $\left.\omega \in{ }^{*} P-P\right)$ such that $a_{n}$ is infinitesimal for all $n$ smaller than $\omega$.

$\left\{a_{n}\right\}$ is called admissible in ${ }^{*} C$ if the relation representing $\left\{a_{n}\right\}$ belongs to the set of relations which are admissible in the sense explained above. Admissible operators, etc., are defined in a similar way. 1.3. shows that the sequence $\left\{a_{n}\right\}$ which is defined by $a_{n}=0$ for finite $n$ and by $a_{n}=1$ for infinite $n$ is not admissible in ${ }^{*} C$.

2. Nonstandard Hilbert space. The selected representation of the Hilbert space $H$ consists of all sequences $\left\{s_{n}\right\}$ of complex numbers such that $\|\sigma\|^{2}=\sum_{n=1}^{\infty}\left|s_{n}\right|^{2}$ converges. The corresponding space ${ }^{*} H$ over ${ }^{*} C$ consists of all admissible sequences $\left\{s_{n}\right\}$ in ${ }^{*} C$ such that $\|\sigma\|^{2}=\sum_{n=1}^{\infty}\left|s_{n}\right|^{2}$ converges, i.e., such that it satisfies the formal (classical) definition of convergence in $L$.

Among the points of ${ }^{*} H$ are the extensions of points of $H$ (as sequences). We identify the points of $H$ with their extension in ${ }^{*} H$ and may then regard $H$ as a subset (though not an admissible subset) of ${ }^{*} H$.

A point $\sigma$ of ${ }^{*} H$ is called norm-finite if $\|\sigma\|$ is a finite real number in the sense explained in section $1 . \sigma$ is near-standard if $\left\|\sigma-\sigma^{0}\right\|$ is infinitesimal for some ${ }^{0} \sigma \in H$. If such a ${ }^{0} \sigma$ exists then it is determined uniquely by $\sigma$. It is called the standard part of $\sigma$.

Applying 1.2. to the partial sums of any point $\sigma=\left\{s_{n}\right\}$ in $H$, we obtain: 
Theorem 2.1. For any $\sigma=\left\{s_{n}\right\}$ in $H$ and any infinite positive integer $\omega$, the sum $\sum_{n=\omega}^{\infty}\left|s_{n}\right|^{2}$ is infinitesimal.

Next, we sketch the proof of:

THEOREM 2.2. A point $\sigma=\left\{s_{n}\right\}$ in ${ }^{*} H$ is near-standard if and only if it is norm-finite and if at the same time $\sum_{n=\omega}^{\infty}\left|s_{n}\right|^{2}$ is infinitesimal for all infinite $\omega$.

Suppose that $\left\|\sigma-{ }^{0} \sigma\right\|$ is infinitesimal for some ${ }^{0} \sigma$ in $H$. Then $\sigma\|=\| \sigma-{ }^{0} \sigma+{ }^{0} \sigma\|\leqq\| \sigma-{ }^{0} \sigma\|+\|{ }^{0} \sigma\|<1+\|{ }^{0} \sigma \|$ so that $\sigma$ is norm-finite. Also, let ${ }^{0} \sigma=\left\{s_{n}^{\prime}\right\}$, then $\sum_{n=\omega}^{\infty}\left|s_{n}^{\prime}\right|^{2}$ is infinitesimal for infinite $\omega$, by 2.1. Also, $\sum_{n=\infty}^{\infty}\left|s_{n}-s_{n}^{\prime}\right|^{2}$ is infinitesimal since this sum cannot exceed $\left\|\sigma-{ }^{0} \sigma\right\|^{2}$. But

$$
\sum_{n=\omega}^{\infty}\left|s_{n}\right|^{2} \leqq\left(\left(\sum_{n=\omega}^{\infty}\left|s_{n}-s_{n}^{\prime}\right|^{2}\right)^{1 / 2}+\left(\sum_{n=\omega}^{\infty}\left|s_{n}^{\prime}\right|^{2}\right)^{1 / 2}\right)^{2},
$$

showing that the conditions of 2.2 are necessary.

Supposing that they are satisfied, $\|\sigma\|$ is finite, hence $\left|s_{n}\right|$ is finite for any $n$ and $s_{n}$ possesses a standard part, ${ }^{0} s_{n}$. Consider the sequence $\left\{s_{n}\right\}$ in $C$. It can be shown that $\sum_{n=1}^{\infty}\left|{ }^{0} s_{n}\right|^{2}$ converges in $C$ and hence, represents a point $\sigma^{\prime}$ in $H$ and ${ }^{*} H$. Thus, if $\sigma^{\prime}=\left\{s_{n}^{\prime}\right\}$ then $s_{n}^{\prime}={ }^{0} s_{n}$ for finite $n$ but not necessarily for infinite $n$. Since, for all finite $k$, $\sum_{n=1}^{k}\left|s_{n}-s_{n}^{\prime}\right|^{2}=\sum_{n=1}^{k}\left|s_{n}-{ }^{0} s_{n}\right|^{2}$ is infinitesimal, it follows from 1.3 that $\sum_{n=1}^{k}\left|s_{n}-s_{n}^{\prime}\right|^{2}$ is still infinitesimal for some infinite $k, k=\omega-1$, say. On the other hand, $\sum_{n=\omega}^{\infty}\left|s_{n}\right|^{2}$ is infinitesimal by assumption, and $\sum_{n=\omega}^{\infty}\left|s_{n}^{\prime}\right|^{2}$ is infinitesimal, by 2.1. The inequality

$$
\begin{aligned}
\left\|\sigma-\sigma^{\prime}\right\|^{2}=\sum_{n=1}^{\infty} \mid s_{n}- & \left.s_{n}^{\prime}\right|^{2} \leqq \sum_{n=1}^{\omega-1}\left|s_{n}-s_{n}^{\prime}\right|^{2} \\
& +\left(\left(\sum_{n=\omega}^{\infty}\left|s_{n}\right|^{2}\right)^{1 / 2}+\left(\sum_{n=\omega}^{\infty}\left|s_{n}^{\prime}\right|^{2}\right)^{1 / 2}\right)^{2},
\end{aligned}
$$

then shows that $\left\|\sigma-\sigma^{\prime}\right\|$ is infinitesimal, $\sigma$ is near-standard with standard part ${ }^{0} \sigma=\sigma^{\prime}$.

The following theorem is proved in [7] for general topological spaces but under somewhat different conditions.

THEOREM 2.3. Let $A$ be a compact set of points in $H$. Then all points of ${ }^{*} A$ (i.e., of the set which corresponds to $A$ in ${ }^{*} H$ ) are near-standard.

Indeed, suppose that $A$ is compact but that $\sigma \in^{*} A$ is not nearstandard. Then there exists a standard positive $r$ such that $\|\sigma-\tau\|>r$ for all $\tau \in H$. This is trivial if $\sigma$ is not norm-finite. If $\sigma$ is norm- 
finite, then by 2.2 , there exists an infinite positive integer $\omega$ such that $\sum_{n=\omega}^{\infty}\left|s_{n}\right|^{2}>2 r^{2}$ for some standard positive number $r$. For any $\tau=\left\{t_{n}\right\}$ in $H, \sum_{n=\omega}^{\infty}\left|t_{n}\right|^{2}$ is infinitesimal. Hence

$$
\begin{aligned}
\|\sigma-\tau\|=\left(\sum_{n=1}^{\infty}\left|s_{n}-t_{n}\right|^{2}\right)^{1 / 2} & \geqq\left(\sum_{n=\omega}^{\infty}\left|s_{n}-t_{n}\right|^{2}\right)^{1 / 2} \\
& \geqq\left(\sum_{n=\omega}^{\infty}\left|s_{n}\right|^{2}\right)^{1 / 2}-\left(\sum_{n=\infty}^{\infty}\left|t_{n}\right|^{2}\right)^{1 / 2}>r .
\end{aligned}
$$

On the other hand, since $A$ is compact it possesses an $r$-net, i.e., for some finite number of points in $A, \tau_{1}, \cdots, \tau_{m}$, and for all $\xi$ in $A$, $\left\|\xi-\tau_{i}\right\|<r$ for some $i, 1 \leqq i \leqq m$. But, for the specified $\tau_{1}, \cdots, \tau_{m}$, this is a property of $H$ which can be formulated as a sentence of $K$. It follows that for all points $\xi$ of $* A$ also $\left\|\xi-\tau_{i}\right\|<r$ for some $i, 1 \leqq i \leqq m$. This contradiction proves the theorem.

3. Operators in nonstandard Hilbert space. An operator from $H$ into $H$ may be regarded as a relation between elements of $H$, i.e., between sequences of elements of $C$ (which are themselves relations). The corresponding operator in ${ }^{*} H$, which is denoted by the same symbol in $L$, will be denoted here also by $T$. This cannot give rise to any confusion. For if $\tau=T \sigma$ in $H$ then $\tau=T \sigma$ also in ${ }^{*} H$ since $\tau=T \sigma$ can be expressed by a sentence of $K$.

In particular, let $T$ be a bounded linear operator defined on all of $H$. For the assumed representation of $H$ by sequences, $T$ has a matrix representation, $T=\left(a_{j k}\right), j, k=1,2,3, \cdots$. The coefficients of this matrix satisfy the conditions:

$$
\text { 3.1. } \quad \begin{array}{ll}
\sum_{k=1}^{\infty}\left|a_{j k}\right|^{2}<\infty & j=1,2,3, \cdots \\
\sum_{j=1}^{\infty}\left|a_{j k}\right|^{2}<\infty & k=1,2,3, \cdots
\end{array}
$$

In ${ }^{*} H$ these subscripts of $\left(a_{j k}\right)$ vary also over the infinite positive integers. By 3.1 and 2.1., $\sum_{k=\omega}^{\infty}\left|a_{j k}\right|^{2}$ is infinitesimal for infinite $\omega$, provided $j$ is finite. This is not necessarily true for infinite $j$ as shown by the matrix for the identity operator.

THEOREM 3.2. Let $T$ be a completely continuous (compact) linear operator on $H$. Then $T$ maps every norm-finite point in * $H$ on a near-standard point.

Proof. If $\sigma$ is norm-finite then $\|\sigma\|<r$ for some positive $S$-number $r$. The sphere $B=\{\xi \mid\|\xi\|<r\}$ is bounded in $H$ and is mapped by $T$ on a set whose closure, $A$, is compact. If the corresponding sets in 
${ }^{*} H$ are ${ }^{*} B$ and ${ }^{*} A$ respectively then $* B$ contains $\sigma$ (since $\sigma$ satisfies the defining condition of $B$ ) and so ${ }^{*} A$ contains $T \sigma$. But ${ }^{*} A$ contains only near-standard points, by 2.3 , so $T \sigma$ is near-standard, proving 3.2.

In a somewhat different setting [7] the converse of 3.2 is also true.

THEOREM 3.3. If $T=\left(a_{j_{k}}\right)$ is a completely continuous linear operator on $H$, then $a_{j k}$ is infinitesimal for all infinite $k$ ( $j$ finite or infinite).

Proof. For finite $j$, this follows from the fact that $\sum_{n=k}^{\infty}\left|a_{j k}\right|^{2}$ is then infinitesimal. For infinite $j$, define $\sigma=\left\{s_{n}\right\}$ by $s_{n}=0$ for $n \neq k$ and by $s_{k}=1$. Then $\|\sigma\|=1$, so $\tau=\left\{t_{j}\right\}=T \sigma$ must be near-standard, by 3.2, where $t_{j}=\sum_{n=1}^{\infty} a_{j n} s_{n}=a_{j k}$. But then $t_{j}=a_{j k}$ must be infinitesimal for infinite $j$, by 2.2 .

An operator $T=\left(a_{j k}\right)$ will be called almost superdiagonal if $a_{j_{k}}=0$ for $j>k+1, k=1,2,3, \cdots$. This definition depends on the specified basis of $H$.

THEOREM 3.4. Let $T$ be a bounded linear operator on $H$ which is almost superdiagonal. Let

3.5 .

$$
p(z)=c_{0}+c_{1} z+\cdots+c_{m} z^{m}, c_{m} \neq 0, m \geqq 1
$$

be a polynomial with standard complex coefficients such that $p(T)$ is completely continuous. Then there exists an infinite positive integer $\omega$ such that $a_{\omega+1, \omega}$ is infinitesimal.

Proof. Put $Q=\left(b_{j k}\right)=p(T)$. We show by direct computation that, for any $h \geqq 1$,

3.6 .

$$
b_{h \div m, h}=c_{m} a_{h+1, h} a_{h+2, h+1} a_{h \div 3, h \div 2} \cdots a_{h+m, h+m-1} \text {. }
$$

By $3.3, b_{h+m, h}$ is infinitesimal for all infinite $h$. Since $c_{m}$ is not infinitesimal, one of the remaining factors on the right hand side of 3.6 must be infinitesimal, e.g, $a_{h+j+1, h \div j}, 0 \leqq j<m$. Setting $\omega=h+j$, we obtain the theorem.

4. Projection operators. Let $E$ be any admissible closed linear subspace of $* H$ within the nonstandard model under consideration. The corresponding projection operator, which reduces to the identity on $E$, will be denoted by $P_{E}$. Given $E$, we define a subset ${ }^{\circ} E$ of $H$ as follows. For any $\sigma \in H, \sigma \in{ }^{\circ} E$ if and only if $\left\|\sigma-\sigma^{\prime}\right\|$ is infinitesimal for some $\sigma^{\prime} \in E$. Since, by a familiar property of projection operatcrs, $\left\|\sigma-\sigma^{\prime}\right\| \geqq\left\|\sigma-P_{E} \sigma\right\|$, it follows that $\sigma \in{ }^{\circ} E$ if and only if $\| \sigma-P_{E} \sigma_{\|}$ is infinitesimal. In that case, $\sigma={ }^{\circ}\left(P_{E} \sigma\right)$. More generally, if $\tau$ is a 
near-standard element of $E$ then ${ }^{\circ} \tau \in{ }^{\circ} E$.

The tools developed so far suffice to establish the following theorem, 4.1, as well as the subsequent theorems, 4.2 ond 4.3.

THEOREM 4.1. Given $E$ as above, the set ${ }^{\circ} E$ is a closed linear subspace of $H$.

Proof. Let $\sigma_{1}, \sigma_{2}$ be elements of ${ }^{\circ} E$. There exist elements $\tau_{1}, \tau_{2}$ of $E$ such that $\| \sigma_{1}-\tau_{1}:$ and $\left\|\sigma_{2}-\tau_{2}\right\|$ are infinitesimal. Then $\tau_{1}+\tau_{2}$ belongs to $E$ and

$$
\left\|\left(\sigma_{1}+\sigma_{2}\right)-\left(\tau_{1}+\tau_{2}\right)\right\| \leqq\left\|\sigma_{1}-\tau_{1}\right\|+\left\|\sigma_{2}-\tau_{2}\right\|
$$

so that the left hand side of this inequality also is infinitesimal. Hence, $\sigma_{1}+\sigma_{2}$ belongs to ${ }^{\circ} E$. Again for $\sigma \in{ }^{\circ} E$ and $\lambda$ standard complex, there exists $\tau \in E$ such that $\|\sigma-\tau\|$ is infinitesimal. Then $\lambda \tau \in E$ and $\|\lambda \sigma-\lambda \tau\|=|\lambda|\|\sigma-\tau\|$ is infinitesimal and so $\lambda \sigma \in{ }^{\circ} E$. This shows that ${ }^{\circ} E$ is linear in the algebraic sense.

Now let $\sigma_{n} \rightarrow \sigma$, where the $\sigma_{n}$ are defined for standard natural $n$ and belong to ${ }^{\circ} E$, and $\sigma$ belongs to $H$. In order to prove that ${ }^{\circ} E$ is closed we have to show that $\sigma$ belongs to ${ }^{\circ} E$. By assumption, the distances $\left\|\sigma_{n}-P_{E} \sigma_{n}\right\|$ are infinitesimal for all $n \in N$. Hence, by Theorem 1.3 there exists an infinite natural number $\omega$ such that $\left\|\sigma_{n}-P_{E} \sigma_{n}\right\|$ is infinitesimal for all $n<\omega$. The sequence of points $\left\{\sigma_{n}\right\}$ in ${ }^{\circ} E \subseteq H$ extends, in ${ }^{*} H$, to a sequence of points defined for all $n \in{ }^{*} N$. Moreover, by 1.2 above, the fact that $\sigma_{n} \rightarrow \sigma$ in $H$ implies that $\left\|\sigma_{n}-\sigma\right\|$ is infinitesimal for all infinite $n$. Hence, for all infinite $n$ less than $\omega,\left\|\sigma-P_{E} \sigma_{n}\right\|$, which does not exceed

$$
\left\|\sigma-\sigma_{n}\right\|+\left\|\sigma_{n}-P_{E} \sigma_{n}\right\|,
$$

also must be infinitesimal. But $P_{E} \sigma_{n} \in E$ and so $\sigma \in{ }^{\circ} E$, as required. This completes the proof of 4.1 .

Let $\omega$ be an infinite natural number. The closed linear subspace of $* H$ which consists of all points $\sigma=\left\{s_{n}\right\}$ such that $s_{n}=0$ for $n>\omega$ will be denoted by $H_{\omega}$. The corresponding projection operator, which will be denoted by $P$ maps any $\sigma=\left\{s_{n}\right\}$ in ${ }^{*} H$ into the point $\sigma^{\prime}=\left\{s_{n}^{\prime}\right\}$, where $s_{n}^{\prime}=s_{n}$ for $n \leqq \omega$ and $s_{n}^{\prime}=0$ for $n>\omega$. For any point $\sigma \in H$, $\|\sigma-P \sigma\|=\left(\sum_{n=\omega+1}^{\infty}\left|s_{n}\right|^{2}\right)^{1 / 2}$ is infinitesimal, by 2.1.

For any bounded linear operator $T$ on $H$ let $T^{\prime}=P T P$, and let $T_{\omega}$ be the restriction of $T^{\prime}$ to $H_{\omega}$. Then $\left\|T^{\prime}\right\| \leqq\|P\|^{2}\|T\| \leqq\|T\|$ and so $\left\|T_{\omega}\right\| \leqq\|T\|$.

THEOREM 4.2. Let $E$ be an admissible closed linear subspace of $H_{\omega}$ which is invariant for $T_{\omega}$, i.e., $T_{\omega} E \subseteq E$. Then ${ }^{\circ} E$ is 
invariant for $T, T^{\circ} E \subseteq{ }^{\circ} E$.

Proof. Let $\sigma \in{ }^{\circ} E$, then we have to show that $T \sigma \in{ }^{\circ} E$. By assumption, there exists a $\tau \in E$ such that $\|\sigma-\tau\|$ is infinitesimal. Then $T_{\omega} \tau \in E$, i.e., $P T \tau \in E$. Thus, in order to show that $T \sigma$ is infinitely close to $E$, we only have to establish that the quantity $a=\|T \sigma-P T \tau\|$ is infinitesimal. Now

$$
\begin{aligned}
a & =\|T \sigma-P T \tau\|=\|T \sigma-P T \sigma+P T(\sigma-\tau)\| \\
& \leqq\|T \sigma-P T \sigma\|+\|P\|\|T\|\|\sigma-\tau\|
\end{aligned}
$$

and $\|T\|$ is a standard real number, while $\|P\| \leqq 1$ and $\|\sigma-\tau\|$ is infinitesimal. At the same time $T \sigma$ is a point of $H$ and so the difference $T \sigma-P T \sigma$ is infinitesimal, as shown above. It follows that $a$ is infinitesimal, and this is sufficient for the proof of 4.2.

The number of dimensions of $H_{\omega}$ as defined within the language $L$ is $\omega, d\left(H_{\omega}\right)=\omega$. In this sense, $H_{\omega}$ is "finite-dimensional". Similarly, with every admissible closed linear subspace $E$ of $H_{\omega}$, there is associated a natural number $d(E)$ in ${ }^{*} C$, which may be finite or infinite, and which has the properties of a dimension to the extent to which these can be expressed as sentences of $K$.

THEOREM 4.3. Let $E$ and $E_{1}$ be two admissible closed linear subspaces of $H_{\omega}$ such that $E \subseteq E_{1}$ and $d\left(E_{1}\right)=d(E)+1$. Then ${ }^{\circ} E \subseteq$ ${ }^{\circ} E_{1}$ and any two points of ${ }^{\circ} E_{1}$ are linearly dependent modulo ${ }^{\circ} E$.

Proof. Since $E \cong E_{1}$, it is trivial that ${ }^{\circ} E \subseteq{ }^{\circ} E_{1}$. Now suppose that ${ }^{\circ} E_{1}$ contains two points $\sigma_{1}$ and $\sigma_{2}$ which are linearly independent modulo ${ }^{\circ} E$. Then $\sigma_{1}$ and $\sigma_{2}$ are infinitely close to points $\tau_{1}, \tau_{2}$ of $E_{1}$, respectively. Since the dimension of $E_{1}$ exceeds that of $E$ only by one, there must be a representation

4.4 .

$$
\tau_{2}=\lambda \tau_{1}+\tau
$$

or vice versa, where $\tau \in E$ and $\lambda$ is an element of ${ }^{*} C$. Now if $\lambda$ were infinitesimal (including $\lambda=0$ ) $\tau_{\text {: }}$ would be infinitely close to $E$, and so $\sigma_{2}$ would be infinitely close to $E$ and would belong to ${ }^{\circ} E$. This is contrary to the assumption that $\sigma_{1}$ and $\sigma_{2}$ are linearly independent modulo ${ }^{\circ} E$. If $\lambda$ were infinite, then the relation

$$
\tau_{1}=\lambda^{-1} \tau_{2}-\lambda^{-1} \tau
$$

(in which $\lambda^{-1}$ is infinitesimal and $\lambda^{-1} \tau$ belongs to $E$ ) would show that $\sigma_{1}$ belongs to ${ }^{\circ} E$. Note that both $\tau_{1}$ and $\tau_{\text {a }}$ are norm-finite since they are infinitely close to the standard points $\sigma_{1}$ and $\sigma_{2}$, respectively.

We conclude that $\lambda$ possesses a standard part, ${ }^{\circ} \lambda$, and that ${ }^{\circ} \lambda \neq 0$. 
Also, $\tau=\tau_{2}-\lambda \tau_{1}$ is infinitely close to $\sigma=\sigma_{2}-{ }^{\circ} \lambda \sigma_{1}$, since

$$
\begin{aligned}
\|\tau-\sigma\| & =\left\|\tau_{2}-\lambda \tau_{1}-\left(\sigma_{2}-{ }^{\circ} \lambda \sigma_{1}\right)\right\| \\
& \leqq\left\|\tau_{2}-\sigma_{2}\right\|+|\lambda|\left\|\tau_{1}-\sigma_{1}\right\|+\left|\lambda-{ }^{\circ} \lambda\right|\left\|\sigma_{1}\right\|
\end{aligned}
$$

so that $\|\tau-\sigma\|$ is infinitesimal. It follows that $\sigma$ belongs to ${ }^{\circ} E$ and that $\sigma_{1}$ and $\sigma_{2}$ are linearly dependent modulo ${ }^{\circ} E$. This contradiction proves the theorem.

5. Proof of the main theorem. We are now ready to prove 1.1. To begin with, we work in the standard model, i.e., in an ordinary Hilbert space $H$ over the complex numbers, $C$. Our method, like that of [1] is based on the fact that in a finite-dimensional space, of dimension $\mu$ say, any linear operator possesses a chain of invariant subspaces

$$
\text { 5.1. } \quad E_{0} \subseteq E_{1} \subseteq E_{2} \subseteq \cdots \subseteq E_{\mu}
$$

where $d\left(E_{j}\right)=j, 0 \leqq j \leqq \mu$, so that $E_{0}=\{0\}$.

The proof of 1.1. is trivial [1] unless for every $\sigma \neq 0$ in $H$, the set $A=\left\{\sigma, T \sigma, T^{2} \sigma, \cdots, T^{n} \sigma, \cdots\right\}$ is linearly independent algebraically and generates the entire space. Assuming from now on that this is the case, we choose $\sigma$ such that $\|\sigma\|=1$, and we replace $A$ by an equivalent orthonormal set $B=\left\{\sigma=\eta_{1}, \eta_{2}, \eta_{3}, \cdots \eta_{n}, \cdots\right\}$ by the GramSchmidt method. Then $\left\{\sigma, T \sigma, \cdots, T^{n-1} \sigma\right\}$ and $\left\{\eta_{1}, \eta_{2}, \cdots \eta_{n}\right\}$ are linearly dependent upon each other. We deduce without difficulty that $T$ is almost superdiagonal with respect to the basis $B$. Representing any $\tau \in H$ by the sequence $\left\{t_{n}\right\}$, where $t_{n}=\left(\tau, \eta_{n}\right)$, we may then identify $H$ with the sequence space considered in the preceding sections. Thus, if $T=\left(a_{j k}\right)$ in this representation, then $a_{j k}=0$ for $j>k+1, k=1,2,3, \cdots$ and, passing to ${ }^{*} C$ and $* H$, there exists an infinite positive integer $\omega$ such that $a_{\omega+1, \omega}$ is infinitesimal, by 3.4. $\omega$ will be kept fixed from now on, and for it we consider the space $H_{\omega}$ and the operators $P$ and $T^{\prime}=P T P$ introduced in Section 4 above.

Let $\xi=\left\{x_{i}\right\}$ be any norm-finite element of $* H$. Consider the difference

$$
\left(T P-T^{\prime}\right) \xi=(I-P) T P \xi=\zeta=\left\{z_{n}\right\} .
$$

We obtain by direct computation that $z_{\omega+1}=a_{\omega+1, \omega} x_{\omega}$, and $z_{n}=0$ for $n \neq \omega+1$. Hence $\|\zeta\| \leqq\left|a_{\omega+1, \omega}\right|\|\xi\|$, so that $\zeta$ is infinitesimal. Using the equivalence relation $\tau_{1} \sim \tau_{2}$ for points of ${ }^{*} H$ such that $\left\|\tau_{1}-\tau_{2}\right\|$ is infinitesimal, we have shown that $T P \xi \sim T^{\prime} \xi$, where the points on both sides of this equivalence are norm-finite. We then prove by induction that: 


\section{2. $T^{r} P \xi \sim\left(T^{\prime}\right)^{r} \xi \quad$ for norm-finite $\xi, \quad r=1,2,3, \cdots$.}

The case $r=1$ has just been disposed of. Suppose 5.2 proved for $r-1, r \geqq 2$. Then

$$
T^{r} P \xi \sim T\left(T^{\prime}\right)^{r-1} \xi=T P\left(T^{\prime}\right)^{r-1} \xi \sim T^{\prime}\left(T^{\prime}\right)^{r-1} \xi=\left(T^{\prime}\right)^{r} \xi
$$

where we have made use of the first equivalence for $\left(T^{\prime}\right)^{r-1} \xi$ in place of $\xi$. Applying 5.2 to the monomials of $p(T)$, and taking into account that $P \xi \sim \xi$ for $\xi \in H_{\omega}$, we obtain

\section{3. $p(T) \xi \sim p\left(T^{\prime}\right) \xi$ for norm-finite $\xi$ in $H_{\omega}$.}

Let $T_{\omega}$ be the restriction of $T^{\prime}$ to $H_{\omega}$, as in Section 4. Since $H_{\omega}$ is "finite" more precisely $\omega$-dimensional in the sense of Nonstandard Analysis, there exists a chain of subspaces as in 5.1 with $\mu=\omega$, such that $T_{\omega} E_{j} \subseteq E_{j}, j=0,1,2, \cdots, \omega$. The $E_{j}$ are also linear subspaces of ${ }^{*} H$. They are finite-dimensional, hence closed, in the sense of Nonstandard Analysis, i.e., they satisfy the formal condition of closedness as expressed within the language $L$. Let $P_{j}$ be the projection operator from ${ }^{*} H$ onto $E_{j}, j=0,1,2, \cdots, \omega$, so that $P_{\omega}=P$.

Suppose $p(z)$ is given by 3.5. For any $\xi \neq 0$ in $H, p(T) \xi$ must be different from 0 otherwise $\xi, T \xi, \cdots, T^{n} \xi$ would be linearly dependent, contrary to assumption. Choose $\xi$ in $H$ with $\|\xi\|=1$. Since $\xi \sim P \xi$, $p(T) \xi \sim p(T) P \xi$, so $p(T) P \xi$ is not infinitesimal and by $5.3, p(T) P \xi$ and hence $p\left(T^{\prime}\right) \xi$ is not infinitesimal. Thus, $\left\|p\left(T^{\prime}\right) \xi\right\|>r$ for some standard positive $r$. Consider the expressions

\section{4 .}

$$
r_{j}=\left\|p\left(T^{\prime}\right) \xi-p\left(T^{\prime}\right) P_{j} \xi\right\|, j=0,1,2, \cdots, \omega,
$$

and note that $r_{j} \leqq\left\|p\left(T^{\prime}\right)\right\|\left\|\xi-P_{j} \xi\right\|$. We have $r_{0}=\left\|p\left(T^{\prime}\right) \xi\right\|$ so $r_{0}>r$. Also $\left\|\xi-P_{\omega} \xi\right\|=\|\xi-P \xi\|$ is infinitesimal, hence $r_{\omega}<r / 2$. It follows that there exists a smallest positive integer $\lambda$ with may be finite or infinite, such that $r_{\lambda}<r / 2$ but $r_{\lambda-1} \geqq r / 2$.

With every $E_{j}$, we associate the closed linear subspace ${ }^{\circ} E_{j}$ of $H$ which was defined in Section 4 . Now ${ }^{\circ} E_{\lambda-1}$ cannot coincide with $H$, more particularly, it cannot include $\xi$. For if it did, then $\left\|\xi-P_{\lambda-1} \xi\right\|$ would be infinitesimal, so $r_{\lambda-1}$, which is bounded by $\left\|p\left(T^{\prime}\right)\right\|\left\|\xi-P_{\lambda-1} \xi\right\|$ would be infinitesimal, contrary to the choice of $\lambda$.

On the other hand ${ }^{\circ} E_{\lambda}$ cannot reduce to $\{0\}$. Consider the point $\eta=p\left(T^{\prime}\right) P_{\lambda} \xi . \quad \eta \in E_{\lambda}$ since $P_{\lambda} \xi \in E_{\lambda}$ and $E_{\lambda}$ is invariant under $p\left(T_{\omega}\right)$ and, equivalently, under $p\left(T^{\prime}\right)$. Also, since $P_{\lambda} \xi \in H_{\omega}$,

$$
\eta=p\left(T^{\prime}\right) P_{\lambda} \xi \sim p(T) P_{\lambda} \xi
$$

where the right-hand side is near-standard, by 3.2 , since $P_{\lambda} \xi$ is normfinite and $p(T)$ is completely continuous. It follows that $\eta$ possesses 
a standard part, ${ }^{\circ} \eta$, and that ${ }^{\circ} \eta$ belongs to ${ }^{\circ} E_{\lambda}$. Again, ${ }^{\circ} \eta=0$ would imply that $\eta$ is infinitesimal. Hence, by 5.4

$$
r_{\lambda} \geqq\left\|p\left(T^{\prime}\right) \xi-\right\| p\left(T^{\prime}\right) P_{\lambda} \xi \|>r-\xi
$$

where $\zeta$ is infinitesimal. Hence $r_{\lambda}>r / 2$, contrary to the choice of $\lambda$. We conclude that ${ }^{\circ} E_{\lambda}$ contains a point different from 0 , i.e., ${ }^{\circ} \eta$.

Both ${ }^{\circ} E_{\lambda-1}$ and ${ }^{\circ} E_{\lambda}$ are invariant for $T$, by 4.2 . If neither were a proper invariant subspace of $H$ for $T$ we should have ${ }^{\circ} E_{\lambda-1}=\{0\}$, ${ }^{\circ} E_{\lambda}=H$. But this contradicts 4.3 , proving 1.1 .

\section{REFERENCES}

1. N. Aronszajn and K. T. Smith, Invariant subspaces of completely continuous operators, Annals of Math. 60 (1954), 345-350.

2. P. R. Halmos, A glimpse into Hilbert space, Lectures on Modern Mathematics, Vol. I, New York-London (1963), 1-22.

3. L. Henkin, Completeness in the theory of types, J. Symbolic Logic 15 (1950), 81-91.

4. A. Robinson, Non-standard analysis, Proc. Royal Acad. Sci, Amsterdam, Ser. A 64, 432-440.

5. - Introduction to model theory and to the metamathematics of Algebra, Studies in Logic and the Foundations of Mathematics, Amsterdam, 1963.

6. - On generalized limits and linear functionals, Pacific J. Math. 14 (1964), 269-283.

7. Topics in non-archimedean Mathematics, Proceedings of the Symposium on Model Theory, Berkeley, 1963. (To be published.)

UNIVERSITY OF WISCONSIN

AND

University of California, Los ANgeles 



\section{PACIFIC JOURNAL OF MATHEMATICS}

\section{EDITORS}

\author{
H. SAMmLSON \\ Stanford University \\ Stanford, California \\ R. M. BLUMENTHAL \\ University of Washington \\ Seattle, Washington 98105
}

\author{
*J. DUGUNDJI \\ University of Southern California \\ Los Angeles, California 90007
}

RICHARD ARENS

University of California

Los Angeles, California 90024

\section{E. F. BECKENBACH \\ B. H. NEUMANN \\ ASSOCIATE EDITORS}

\section{SUPPORTING INSTITUTIONS}

\author{
UNIVERSITY OF BRITISH COLUMBIA \\ CALIFORNIA INSTITUTE OF TECHNOLOGY \\ UNIVERSITY OF CALIFORNIA \\ MONTANA STATE UNIVERSITY \\ UNIVERSITY OF NEVADA \\ NEW MEXICO STATE UNIVERSITY \\ OREGON STATE UNIVERSITY \\ UNIVERSITY OF OREGON \\ OSAKA UNIVERSITY \\ UNIVERSITY OF SOUTHERN CALIFORNIA
}

\author{
STANFORD UNIVERSITY \\ UNIVERSITY OF TOKYO \\ UNIVERSITY OF UTAH \\ WASHINGTON STATE UNIVERSITY \\ UNIVERSITY OF WASHINGTON \\ * * * \\ AMERICAN MATHEMATICAL SOCIETY \\ CHEVRON RESEARCH CORPORATION \\ TRW SYSTEMS \\ NAVAL ORDNANCE TEST STATION
}

\footnotetext{
Mathematical papers intended for publication in the Pacific Journal of Mathematics should be typewritten (double spaced). The first paragraph or two must be capable of being used separately as a synopsis of the entire paper. It should not contain references to the bibliography. Manu. scripts may be sent to any one of the four editors. All other communications to the editors should be addressed to the managing editor, Richard Arens at the University of California, Los Angeles, California 90024.

50 reprints per author of each article are furnished free of charge; additional copies may be obtained at cost in multiples of 50 .
}

The Pacific Journal of Mathematics is published monthly. Effective with Volume 16 the price per volume (3 numbers) is $\$ 8.00$; single issues, $\$ 3.00$. Special price for current issues to individual faculty members of supporting institutions and to individual members of the American Mathematical Society: $\$ 4.00$ per volume; single issues $\$ 1.50$. Back numbers are available.

Subscriptions, orders for back numbers, and changes of address should be sent to Pacific Journal of Mathematics, 103 Highland Boulevard, Berkeley 8, California.

Printed at Kokusai Bunken Insatsusha (International Academic Printing Co., Ltd.), No. 6, 2-chome, Fujimi-cho, Chiyoda-ku, Tokyo, Japan.

PUBLISHED BY PACIFIC JOURNAL OF MATHEMATICS, A NON-PROFIT CORPORATION

The Supporting Institutions listed above contribute to the cost of publication of this Journal, but they are not owners or publishers and have no responsibility for its content or policies.

* Paul A. White, Acting Editor until J. Dugundji returns. 


\section{Pacific Journal of Mathematics}

\section{Vol. 16, No. $3 \quad$ BadMonth, 1966}

Gert Einar Torsten Almkvist, Stability of linear differential equations with

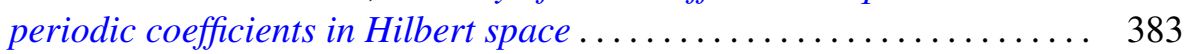

Richard Allen Askey and Stephen Wainger, A transplantation theorem for ultraspherical coefficients ................................ 393

Joseph Barback, Two notes on regressive isols .................. 407

Allen Richard Bernstein and Abraham Robinson, Solution of an invariant subspace problem of K. T. Smith and P. R. Halmos .............. 421

P. R. Halmos, Invariant subspaces of polynomially compact operators . . . . 433

Leon Bernstein, New infinite classes of periodic Jacobi-Perron algorithms.................................... 439

Richard Anthony Brualdi, Permanent of the direct product of matrices .... . 471

W. Wistar (William) Comfort and Kenneth Allen Ross, Pseudocompactness and uniform continuity in topological groups .................. 483

James Michael Gardner Fell, Algebras and fiber bundles . . . . . . . . . . . . 497

Alessandro Figà-Talamanca and Daniel Rider, A theorem of Littlewood and lacunary series for compact groups ..................... 505

David London, Two inequalities in nonnegative symmetric matrices...... 515

Norman Jay Pullman, Infinite products of substochastic matrices ........ 537

James McLean Sloss, Reflection and approximation by interpolation along

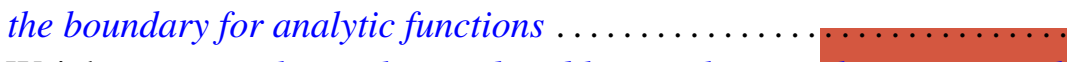

Carl Weinbaum, Visualizing the word problem, with an application to sixth groups................................... 